\title{
Pomegranate for Your Cardiovascular Health
}

\author{
Michael Aviram, D.Sc, ${ }^{*}$ and Mira Rosenblat, M.Sc. \\ The Lipid Research Laboratory, The Rappaport Faculty of Medicine and Research Institute, Technion- \\ Institute of Technology, and Rambam Medical Center, Haifa, Israel
}

\begin{abstract}
Pomegranate is a source of some very potent antioxidants (tannins, anthocyanins) which are considered to be also potent anti-atherogenic agents. The combination of the above unique various types of pomegranate polyphenols provides a much wider spectrum of action against several types of free radicals. Indeed, pomegranate is superior in comparison to other antioxidants in protecting low-density lipoprotein (LDL, "the bad cholesterol") and high-density lipoprotein (HDL, "the good cholesterol") from oxidation, and as a result it attenuates atherosclerosis development and its consequent cardiovascular events. Pomegranate antioxidants are not free, but are attached to the pomegranate sugars, and hence were shown to be beneficial even in diabetic patients. Furthermore, pomegranate antioxidants are unique in their ability to increase the activity of the HDL-associated paraoxonase 1 (PON1), which breaks down harmful oxidized lipids in lipoproteins, in macrophages, and in atherosclerotic plaques. Finally, unique pomegranate antioxidants beneficially decrease blood pressure. All the above beneficial characteristics make the pomegranate a uniquely healthy fruit.
\end{abstract}

KEY WORDS: Atherosclerosis, pomegranate, antioxidant, polyphenols, macrophages, lipoproteins (LDL, HDL)

\begin{abstract}
Abbreviations: AAPH, 2,2'-azobis amidinopropane hydrochloride; ACE, angiotensin-converting enzyme; BP, blood pressure; CAS, carotid artery stenosis; CHD, coronary heart disease; CIMT, carotid intima-media thickness; EDV, enddiastolic velocity; GAE, gallic acid equivalents; HDL, high-density lipoprotein; HMDM, human monocyte-derived macrophages; LDL, low-density lipoprotein; LPDS, lipoprotein-deficient serum; MI, myocardial infarction; Ox-LDL, oxidized LDL; PJ, pomegranate juice; POMxl, an extract of the pomegranate outer peel; PON, paraoxonase; PSV, peak systolic velocity; ROS, reactive oxygen species; TAS, total antioxidant status; TBARS, thiobarbituric acid reactive substances; TGs, triglycerides; VLDL, very-low-density lipoprotein.
\end{abstract}

Citation: Aviram M, Rosenblat M. Pomegranate for Your Cardiovascular Health. RMMJ 2013;4 (2):eoo13. doi:10.5041/RMMJ.10113

Copyright: (C) 2013 Aviram and Rosenblat. This is an open-access article. All its content, except where otherwise noted, is distributed under the terms of the Creative Commons Attribution License (http://creativecommons.org/licenses/by/3.o), which permits unrestricted use, distribution, and reproduction in any medium, provided the original work is properly cited.

Conflict of interest: No potential conflict of interest relevant to this article was reported.

* To whom correspondence should be addressed. E-mail: aviram@tx.technion.ac.il 


\section{POMEGRANATE FRUIT COMPOSITION}

The pomegranate tree, which is said to have flourished in the Garden of Eden, has been extensively used as a folk medicine in many cultures. ${ }^{1}$ Edible parts of pomegranate fruits (about $50 \%$ of the total fruit weight) comprise $80 \%$ juice and 20\% seeds. Pomegranate juice (PJ) consists of the crushed fruit only. Fresh juice contains $85 \%$ moisture, $10 \%$ total sugars, $1.5 \%$ pectin, and also antioxidants such as ascorbic acid and polyphenols. Content of soluble polyphenols in PJ varies within the limits of $0.2 \%-1.0 \%$, depending on the variety, and includes mainly anthocyanins (such as cyanidin-3-glycoside, cyanidin-3,3-diglycoside, and delphindin-3-glycoside) and anthoxantins (such as catechins, ellagic tannins, and gallic and ellagic acids). Ellagic acid and hydrolyzable ellagitannins are both implicated in protection against atherogenesis, along with their potent antioxidant capacity. Punicalagin is the major ellagitannin in PJ, and this compound is responsible for the high antioxidant activity of PJ. ${ }^{2-4}$

\section{POMEGRANATE JUICE BIOAVAILABILITY}

In vivo studies were conducted in order to evaluate whether the active antioxidant components of PJ are absorbed. Recent studies examined the bioavailability and metabolism of punicalagin in the rat as an animal model.5,6 Two groups of rats were studied. One group was fed for 37 days with standard rat diet $(n=5)$, and the second one with the same diet plus $6 \%$ punicalagin $(n=5)$. The daily intake of punicalagin ranged from $0.6 \mathrm{~g}$ to $1.2 \mathrm{~g}$. Glucuronides of methyl ether derivatives of ellagic acid and punicalagin were detected in plasma. $6 \mathrm{H}-$ Dibenzo [b, d] pyran-6-one derivatives were also observed in the plasma, especially during the last few weeks of the study. In urine, the metabolite urolithin was observed along with $6 \mathrm{H}$-dibenzo $[\mathrm{b}, \mathrm{d}]$ pyran-6-one derivatives, and they were present as aglycones or as glucuronides. It was concluded that since only $3 \%-6 \%$ of the ingested punicalagin was detected as such, or as metabolites in urine and feces, the majority of this ellagitannin has to be converted to undetectable metabolites or accumulated in non-analyzed tissues. Only traces of punicalagin metabolites were detected in liver or kidney. In humans, following consumption of PJ (180 mL) containing $25 \mathrm{mg}$ of ellagic acid and 318 mg of hydrolyzable ellagitannins (as punicalagin), ellagic acid was detected in human plasma 1 hour post-ingestion at a maximum concentration of $32 \mathrm{ng} / \mathrm{mL}$, and by 4 hours it was completely eliminated.7

Upon analyzing the influence of the physiological conditions in the stomach and small intestine on pomegranate bioactive compounds bioavailability using an in vitro availability method, 8 it was demonstrated that pomegranate phenolic compounds are available during digestion in a high amount (29\%). Nevertheless, due to $\mathrm{pH}$, anthocyanins are largely transformed into non-red forms, or degraded. Thus, active components of PJ are indeed absorbed and subsequently affect biological processes which are related to atherogenesis protection.

\section{POMEGRANATE CONSUMPTION ATTENUATES ATHEROSCLEROSIS DEVELOPMENT}

PJ is suggested as the "heart-healthy" fruit juice, 9 and it was indeed shown to attenuate cardiovascular diseases..$^{10}$ Measurements of the arterial stiffness of the common carotid arteries in 73 patients with at least one cardiovascular risk factor who consumed PJ (Wonderful variety, $240 \mathrm{~mL} /$ day for 1 year), showed trends to increased arterial elasticity in the PJ-treated group versus the placebo-treated group (who received beverage of similar caloric content, flavor, and color).

The effect of a daily consumption of PJ for 3 months on myocardial perfusion in 45 patients who had coronary heart disease (CHD) was also studied. ${ }^{11}$ Patients were randomly assigned into one of two groups: a PJ group (240 mL/day) or a placebo group. The experimental and control groups showed similar levels of stress-induced ischemia at baseline. After 3 months, however, the extent of stress-induced ischemia decreased in the pomegranate group, but increased in the control group. This benefit was observed without changes in cardiac medications, blood sugar, hemoglobin A1c, body weight, or blood pressure, in either group. ${ }^{11} \mathrm{We}$ next investigated the effects of PJ consumption by patients with carotid artery stenosis (CAS) on carotid lesion size, in association with changes in oxidative stress..$^{12}$ Ten patients were supplemented with PJ for up to 1 year, and nine CAS patients who did not consume PJ served as a control group. Blood samples were collected before treatment and after 3 , 6, 9, and 12 months of PJ consumption. Patients' carotid intima-media thickness (CIMT) was compared between the PJ group and the control 
group. While in the control patients group (no PJ) CIMT increased by $10 \%$ after 1 year (Figure 1A), PJ consumption resulted in a significant CIMT reduction, by up to $35 \%$ (Figure 1B). Analysis of the mean CIMT (of the left and right common carotid arteries) before and during PJ consumption revealed a gradual reduction of $13 \%, 22 \%, 26 \%$, and $35 \%$, as observed after $3,6,9$, and 12 months of PJ consumption, respectively, in comparison to baseline values. On examination of the internal carotid arteries, flow velocities were calculated at the stenotic sites and expressed by peak systolic velocity (PSV) and end-diastolic velocity (EDV). The ultrasound outcome data were the change over time in maximal IMT, which was measured in the same preselected carotid artery segments. Twelve months of PJ consumption resulted in PSV reduction by $12 \%$ and $28 \%$ in the left and the right carotid arteries, respectively. Mean carotid EDV of both left and right carotid arteries gradually decreased, by $16 \%$, $20 \%, 31 \%$, and $44 \%$ after $3,6,9$, and 12 months of PJ consumption, respectively (Figure 1C). ${ }^{12}$

A randomized, double-blind trial assessed the influence of PJ consumption on anterior and posterior CIMT progression rates in subjects at moderate risk for coronary heart disease. Subjects were men (45-74 years old) and women (55-74 years old) with one or more major CHD risk factors and baseline posterior wall CIMT of $0.7-2.0 \mathrm{~mm}$, without any significant stenosis. Participants consumed $240 \mathrm{~mL} /$ day of PJ $(n=146)$, or a control beverage $(n=143)$ for up to 18 months. No significant difference in overall CIMT progression rate was observed between PJ and control treatments. In exploratory analyses, however, of
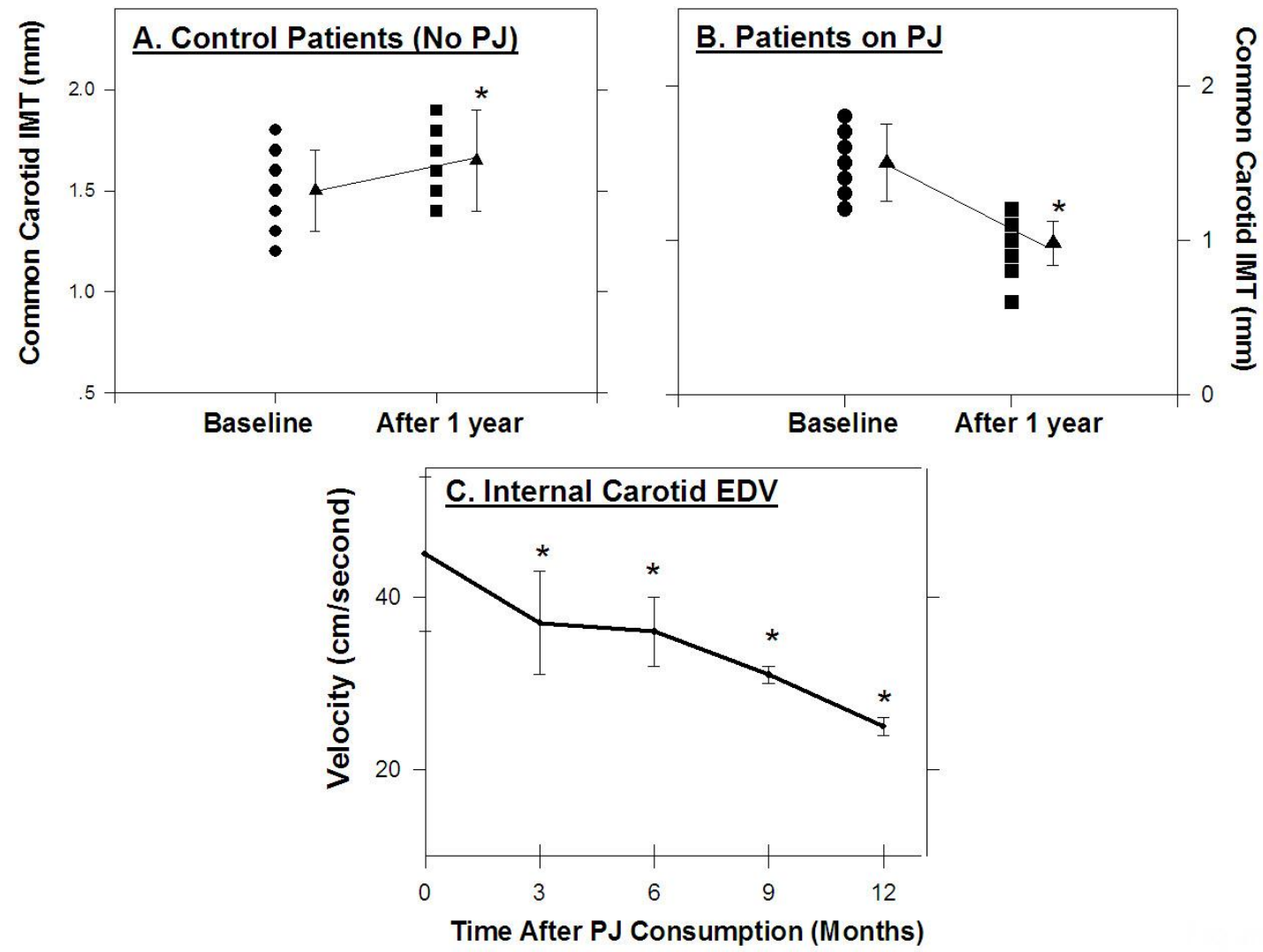

Figure 1. The effect of PJ consumption by patients with CAS on CIMT and on internal carotid EDV.

Ten patients with CAS were supplemented with PJ for up to 1 year. Common CIMT and EDV were measured in the patients' left and right carotid arteries before treatment (baseline) and during PJ consumption. The mean values of both carotid arteries are presented. The individual results of mean common carotid artery IMT, as well as the averages of all mean values $\pm S E M$, are shown $(A$ and $B$ ). The averages of all mean values \pm SEM are shown for internal carotid artery EDV (C). * $P<0.01$ (after PJ consumption versus Baseline-“0” time). 
subjects in the most adverse tertiles for baseline serum lipid peroxides, triglycerides (TGs), highdensity lipoprotein (HDL) cholesterol, TGs/HDL cholesterol, total cholesterol/HDL cholesterol, and apolipoprotein-B100, those in the PJ group had significantly less anterior wall and/or composite CIMT progression versus control subjects. These results suggest that, in subjects at moderate $\mathrm{CHD}$ risk, PJ consumption had no significant effect on overall CIMT progression rate, but slowed CIMT progression in subjects with increased oxidative stress and disturbances in the TG-rich lipoprotein/ HDL axis. ${ }^{13}$

\section{INHIBITORY EFFECT OF POMEGRANATE CONSUMPTION ON SERUM LIPID PEROXIDATION}

The oxidative modification hypothesis of atherosclerosis proposes that low-density lipoprotein (LDL) oxidation plays a pivotal role in early atherogenesis. This hypothesis is supported by evidence that oxidized LDL (Ox-LDL) is present in atherosclerotic lesions, and in human plasma from patients with cardiovascular diseases, and it correlates with the presence of angiographically documented complicated plaques, ${ }^{14-17}$ thus identifying those patients who are at increased risk for future myocardial infarction (MI), independently of other risks. Since PJ contains very potent antioxidants, it can attenuate atherosclerosis development by reducing oxidative stress in these patients. Indeed, human plasma obtained from healthy subjects after 2 weeks of PJ consumption (50 mL PJ concentrate/day, equivalent to $1.5 \mathrm{mmol}$ total polyphenols) demonstrated a small but significant $(P<0.01) 16 \%$ decreased susceptibility to free radical-induced lipid peroxidation, in comparison to plasma obtained prior to PJ consumption, as measured by lipid peroxides formation, or by total antioxidant status (TAS) in serum. ${ }^{18}$ To determine the effect of increasing or decreasing the dosages of PJ on plasma lipid peroxidation, and to analyze the capability of PJ to maintain its effect after termination of juice consumption, three subjects were further studied. Supplementation of $20 \mathrm{~mL}$ of PJ concentrate/day for one week resulted in a significant decrease of $11 \%$ in plasma lipid peroxidation, compared to plasma obtained prior to PJ consumption. Supplementation of $50 \mathrm{~mL}$ PJ concentrate/day for one more week exhibited a further $21 \%$ decrease in plasma lipid peroxidation. However, a further increase in the supplemented PJ to $80 \mathrm{~mL}$ of PJ concentrate/day for an additional one week did not further inhibit plasma susceptibility to lipid peroxidation. Gradual decreasing of the PJ dosage in these three subjects down to $40 \mathrm{~mL} /$ day for one week, and then to $20 \mathrm{~mL} /$ day for an additional two weeks, did not significantly affect plasma lipid peroxidation, which remained low in comparison to the levels obtained after supplementation of $80 \mathrm{~mL}$ of PJ concentrate/day. Two weeks after cessation of PJ supplementation the reduced rate of plasma susceptibility to lipid peroxidation was sustained. After a further four weeks with no PJ consumption, plasma lipid peroxidation returned to the higher values obtained before PJ consumption. ${ }^{18}$

The effect of PJ consumption by patients with CAS on their serum oxidative state was also measured. ${ }^{12}$ A significant $(P<0.01)$ reduction in the concentration of antibodies against Ox-LDL by $24 \%$ and $19 \%$ was observed after 1 and 3 months of PJ consumption, respectively (from $2070 \pm 61 \mathrm{EU} / \mathrm{mL}$ before treatment to $1563 \pm 69$ and $1670 \pm 52 \mathrm{EU} / \mathrm{mL}$ after 1 and 3 months of PJ consumption, respectively). TAS in serum from these patients was substantially increased 2.3-fold (from $0.95 \pm 0.12$ $\mathrm{nmol} / \mathrm{L}$ at baseline, up to $2.20 \pm 0.25 \mathrm{nmol} / \mathrm{L}$ after 12 months of PJ consumption). These results indicate that PJ administration to patients with CAS substantially reduced their serum oxidative status and could thus inhibit plasma lipid peroxidation. The susceptibility of the patients' serum to free radical-induced oxidation decreased after 12 months of PJ consumption by up to 62\% (Figure 2A). Increased oxidative stress was observed in the serum of non-insulin-dependent type 2 diabetes mellitus patients versus healthy subjects (Figure 2B). Consumption of $50 \mathrm{~mL}$ of PJ per day for a period of 3 months resulted in a significant reduction in the basal serum thiobarbituric acid reactive substances (TBARS) levels, by $28 \%$ (Figure 2B).19 Consumption of PJ for 1 and 2 weeks by healthy volunteers increased the resistance of their LDL to copper ion-induced oxidation, as shown by a prolongation of the lag time required for the initiation of LDL oxidation, by $29 \%$ and $43 \%$, in comparison to LDL obtained prior to juice consumption. Similarly, the resistance of their HDL to copper ion-induced oxidation also gradually increased after PJ consumption, as shown by a prolongation in the lag time required for the initiation of HDL oxidation from $37 \pm 2$ minutes to $45 \pm 6$ minutes before and 2 weeks after PJ consumption, respectively. ${ }^{18} \mathrm{PJ}$ consumption by patients with CAS 
resulted in a significant reduction in the basal level of LDL-associated lipid peroxides by $43 \%$, $89 \%$, $86 \%$, and $90 \%$ after $3,6,9$, and 12 months of PJ consumption, respectively, and, in parallel, it increased the resistance of LDL to copper ioninduced oxidation. This was demonstrated by reduced formation of lipid peroxides in LDL during its incubation with copper ions (by 40\%, 49\%, 57\%, and $59 \%$ after $3,6,9$, and 12 months of PJ consumption, respectively). ${ }^{12}$

\section{THE STIMULATORY EFFECT OF POMEGRANATE CONSUMPTION ON SERUM PARAOXONASE 1 (PON1)}

Most of the serum antioxidant and anti-atherogenic enzyme, PON1, is HDL-associated. ${ }^{20}$ Still, low levels of PON1 are also associated with chylomicrons and with very-low-density lipoprotein (VLDL), but not with LDL. ${ }^{21}$ PON1 has a protective role in the attenuation of cardiovascular diseases. ${ }^{22}$ Serum
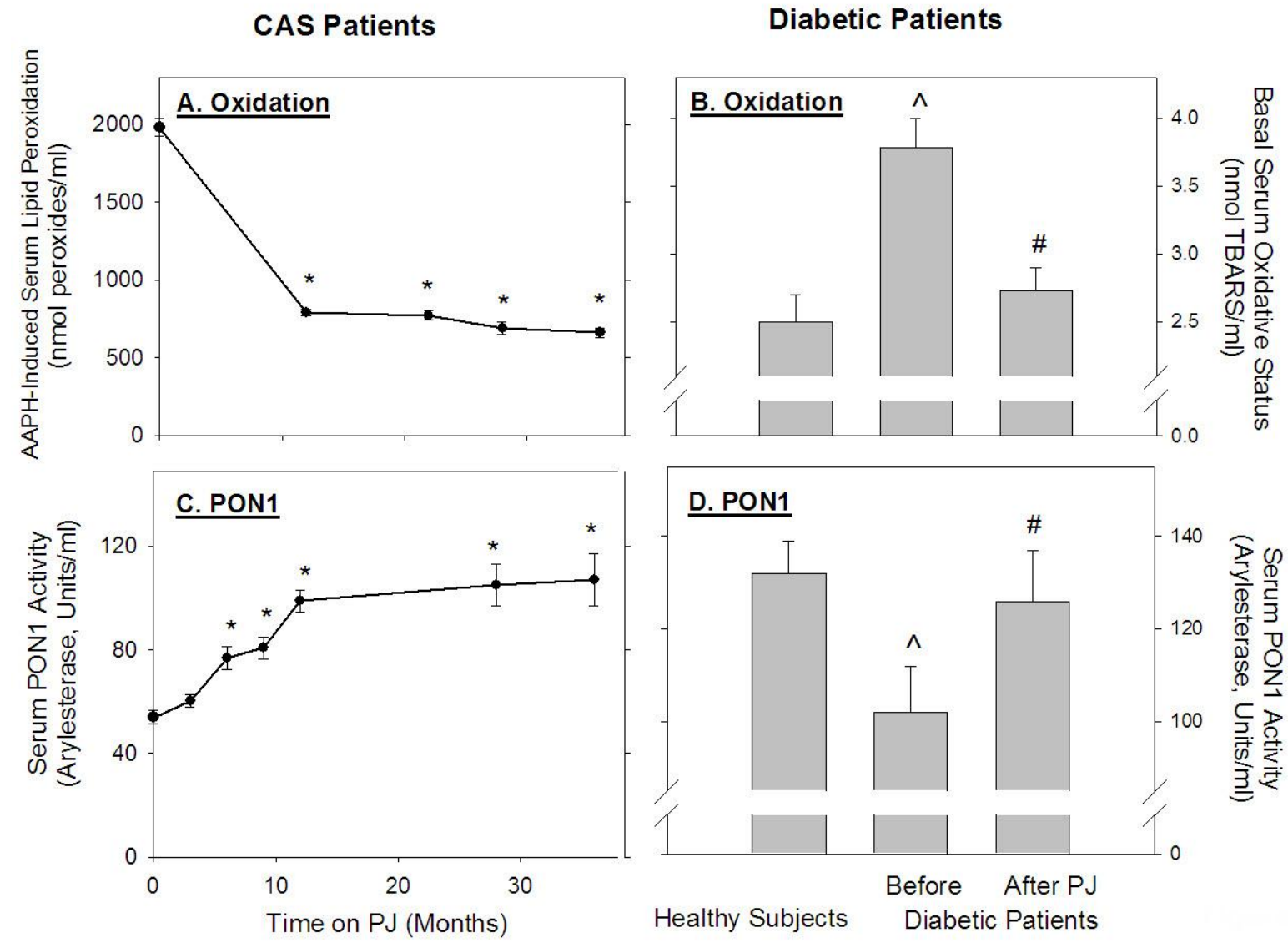

Figure 2. The effect of PJ consumption by patients with CAS, or by diabetic patients, on their serum oxidative stress and on serum PON1 activity.

A and C: Ten patients with CAS were supplemented with PJ for a 1-year period. Blood samples were drawn from the patients before, and after 3, 6, 9, and 12 months of PJ consumption. B and D: Ten patients with type 2 diabetes mellitus were supplemented with PJ for 3 months. Blood samples were drawn from the patients before, and after PJ consumption and also from 10 healthy subjects.

A: The serum susceptibility to oxidation by 2,2 '-azobis amidinopropane hydrochloride (AAPH) was determined by the lipid peroxides assay. B: Basal serum oxidative status was determined by the level of thiobarbituric acid reactive substances (TBARS). C and D: Serum PON1 arylesterase activity was measured using phenyl acetate as the substrate. Results are expressed as mean $\pm \mathrm{SD}(n=10) .{ }^{*} P<0.01$ (after $\mathrm{PJ}$ consumption versus before treatment), ${ }^{\wedge} P<0.01$ (diabetic patients before versus healthy subjects), \#P<0.01 (diabetic patients after PJ versus before). 
PON1 concentration and activity are better predictors of the risk for cardiovascular diseases than the PON1 genotype. ${ }^{23}$ A negative association was observed between serum PON1 activity and IMT in subjects with CHD. ${ }^{24}$ Attenuation of atherosclerosis by PON1 can result from its ability to hydrolyze specific oxidized lipids in lipoproteins, ${ }^{25}$ in arterial wall cells (including macrophages), ${ }^{26,27}$ and in atherosclerotic lesions. ${ }^{28}$

The increased resistance of LDL and of HDL to oxidation after PJ administration to healthy subjects, or to patients with CAS, could have also resulted from increased serum HDL-associated PON1 activity. Indeed, a significant $18 \%$ increase in serum PON1 activity was monitored in healthy subjects after PJ consumption for a period of 2 weeks. ${ }^{18}$ In CAS patients, serum PON1 arylesterase activity significantly increased by $11 \%, 42 \%, 49 \%$, and $83 \%$ after $3,6,9$, and 12 months of PJ consumption, respectively (Figure $2 \mathrm{C}$ ), ${ }^{12}$ and in patients with type 2 diabetes mellitus it significantly increased by $12 \%$ after PJ consumption for 3 months (Figure 2D). ${ }^{19}$

The increment in PON1 protein could result from the direct effect of PJ on PON1 expression in the liver. ${ }^{29}$ The PJ-induced increment in PON1 activity could also result from the reduction in oxidative stress, since oxidized lipids inactivate PON1.30 In addition, association of PON1 with HDL stabilizes the enzyme and stimulates its lactonase activity. ${ }^{20} \mathrm{In}$ diabetic patients, PON1 dissociates from HDL, and as a consequence, it is less biologically active. ${ }^{1} \mathrm{We}$ thus investigated the effects of PJ and POMxl (an extract of the pomegranate outer peel) consumption on PON1 association with HDL in diabetic patients. ${ }^{32}$ HDL-associated PON1 arylesterase and lactonase activities increased significantly after PJ consumption, by $34 \%-45 \%$, as compared to the baseline levels (Figure 3). In male patients who consumed POMxl, and in female patients who consumed PJ, a similar pattern was observed, although to a lesser extent. PON1 protein binding to HDL was significantly increased by $32 \%$ following PJ consumption, while the level of PON1 in the lipoprotein-deficient serum (LPDS) decreased by $62 \%$, suggesting that PJ consumption resulted in increased free PON1 binding to the HDL. A similar trend of increased PON1 protein association with HDL was observed in males following POMxl consumption, as after 4 weeks of POMxl consumption HDL-bound PON1 protein increased by $17 \%$ compared to baseline values. The above results were confirmed also in in vitro study where serum from diabetic patients was incubated with PJ or with punicalagin, or with no addition (control), for 2 hours at $37^{\circ} \mathrm{C}$. Then, HDL was isolated from the serum by ultracentrifugation, and Western blot analysis was performed. After serum incubation with PJ (18 $\mu$ g gallic acid equivalents (GAE)/mL) or with punicalagin, the protein content of HDL-bound PON1 significantly increased by $36 \%$ and by $14 \%$, respectively, as compared to control serum. Upon increasing the concentration of $\mathrm{PJ}$ or punicalagin up to $36 \mu \mathrm{g} \mathrm{GAE} / \mathrm{mL}$, HDL-bound PON1 protein further increased, and it was $62 \%$ or $83 \%$ higher than that observed in control patients' serum (no PJ), respectively.

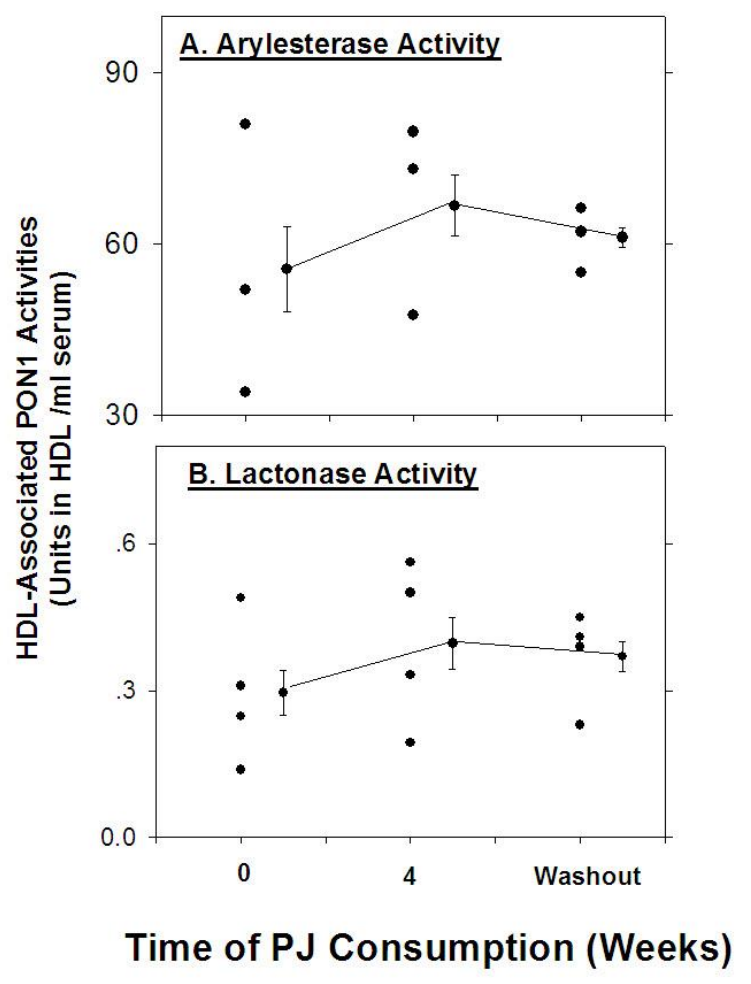

Figure 3. The effect of $\mathrm{PJ}$ consumption by diabetic males on their HDL-associated PON1 activities.

Ten male patients with type 2 diabetes mellitus consumed $50 \mathrm{~mL}$ of concentrated pomegranate juice (PJ) per day (which contain $1.5 \mathrm{mmol}$ of total polyphenols) for a period of 1 month, followed by a period of 4 weeks' "wash-out." Blood samples were collected from the patients before ( 0 time) and 4 weeks after PJ consumption, as well as after the "wash-out" period. The HDL fractions were isolated from the blood samples of four patients by ultracentrifugation. The HDLassociated PON1 arylesterase (A), and lactonase activities (B) were determined. The individual results, as well as the mean $\pm S D$ are shown. 
We thus conclude that PJ as well as POMxl consumption by diabetic patients contributes to PON1 stabilization by increasing its association with HDL and therefore enhancing PON1 catalytic activities. The ratio between HDL-associated PON1 and free PON1 gradually decreased as the extent of HDL oxidation increased. The antioxidants vitamin E or PJ inhibited the oxidation-mediated redistribution of PON1 in serum. Indeed, PJ and its purified major polyphenols punicalagin, gallic acid, and ellagic acid all increased PON1 binding also to HDL.33 Furthermore, PON1 associated more efficiently with HDLs isolated from diabetic patients after PJ consumption versus the patients' HDL isolated prior to $\mathrm{PJ}$ consumption. 33

\section{THE INHIBITORY EFFECT OF POMEGRANATE CONSUMPTION ON BLOOD PRESSURE}

As some antioxidants were recently shown to reduce blood pressure (BP), we studied the effect of PJ consumption ( $50 \mathrm{~mL}, 1.5 \mathrm{mmol}$ of total polyphenols per day, for 2 weeks) by hypertensive patients on their BP and on serum angiotensin-converting enzyme (ACE) activity. ${ }^{34} \mathrm{~A}$ 36\% decrement in serum ACE activity and a $5 \%$ reduction in systolic BP were noted. A similar dose-dependent inhibitory effect (31\%) of PJ on serum ACE activity was observed also in vitro. As reduction in serum ACE activity, even with no decrement in blood pressure BP, was previously shown to attenuate atherosclerosis, PJ can offer a wide protection against cardiovascular diseases which could be related to its inhibitory effect on oxidative stress and on serum ACE activity.

In CAS patients the systolic BP was significantly $(P<0.05)$ reduced by $7 \%, 11 \%, 10 \%, 10 \%$, and $12 \%$ after $1,3,6$, 9, and 12 months of PJ consumption, respectively, compared to values obtained before treatment. In contrast, PJ consumption had no significant effect on the patient's diastolic blood pressure. ${ }^{12}$

In another study, healthy participants consumed $330 \mathrm{~mL} /$ day of PJ or control drink for 4 weeks. 35 Measurements were made at baseline and at 4 weeks. There was a significant fall in systolic BP $(-3.14 \mathrm{mmHg}, P<0.001)$, diastolic BP $(-2.33$ $\mathrm{mmHg}, P<0.001$ ), and mean arterial pressure $(-2.60 \mathrm{mmHg}, P<0.001)$. The fall in BP was not paralleled by changes in concentration of serum ACE.
The effect of PJ supplementation for a short term was also analyzed. ${ }^{36}$ Nineteen young, healthy men completed a randomized, controlled cross-over trial. The active drink (containing a pomegranate extract) was consumed during a high-fat meal (ET-DUR) or 15 min before (ET-PRE), and the placebo drink (no pomegranate extract) was consumed during the high-fat meal (control). Postprandial lipemia was assessed by venous plasma triglyceride concentration. Blood pressure and digital volume pulse, to measure reflection index (DVP-RI) and stiffness index (DVP-SI), were monitored at baseline and at 2 and 4 hours. Systolic BP increased in the ET-PRE and ET-DUR groups to a lesser extent than in the control group (treatment effect $P=0.041$ ). There were no treatment effects for DVP-RI, DVP-SI, or diastolic BP. In conclusion, consumption of a single drink containing ellagitannin-rich pomegranate extract did not decrease postprandial plasma triglyceride concentrations, but suppressed the postprandial increase in systolic BP following the high-fat meal. ${ }^{36}$ More clinical research is needed as a number of the studies discussed include small sample sizes and few studies seem to have been undertaken in the recent $5-10$ years. 37

\section{THE INHIBITORY EFFECT OF POMEGRANATE CONSUMPTION ON MACROPHAGE ATHEROGENICITY}

Macrophage cholesterol, triglyceride, and oxidized lipids accumulation and foam cell formation are the hallmarks of early atherogenesis. ${ }^{38-40}$ Cholesterol accumulation in macrophages can result from impaired balance between external and internal cholesterol sources. LDL is oxidized in vivo by arterial wall cells.41,42 Ox-LDL is taken up by macrophages at an enhanced rate via scavenger receptors which, unlike the LDL receptor, are not down-regulated by intracellular cholesterol content and therefore lead to accumulation of cholesterol in the cells. The cellular cholesterol levels are determined also by the cholesterol biosynthesis rate and by the rate of HDL-mediated cholesterol efflux. We have demonstrated increased oxidative stress in human monocyte-derived macrophages (HMDM) isolated from patients with type 2 diabetes mellitus versus healthy subjects (Figure 4A). After consumption of PJ for 3 months, the patients' HMDM produced less reactive oxygen species (ROS) in comparison to HMDM before PJ consumption (Figure 4A), respectively. Similarly, incubation of 
the human carotid lesions from CAS patients with LDL (100 $\mu \mathrm{g}$ of protein $/ \mathrm{mL}$ ) for 18 hours under oxidative stress (in the presence of copper ions), revealed that PJ consumption for 3 or 12 months resulted in $43 \%$ and $32 \%$ reduced capacity of the lesion to oxidize LDL, respectively (Figure 4B). The reduction in cellular oxidative stress can result from PJ-induced increment in PON1, and/or from PJ- induced increment in paraoxonase 2 (PON2).43 PON2 is expressed in arterial wall cells including macrophages, 44 and it protects the cells from oxidative stress and apoptosis. 45

PJ consumption by patients with type 2 diabetes mellitus significantly decreased the extent of OxLDL cellular uptake by their HMDM (by $36 \%$,
Diabetic Patients
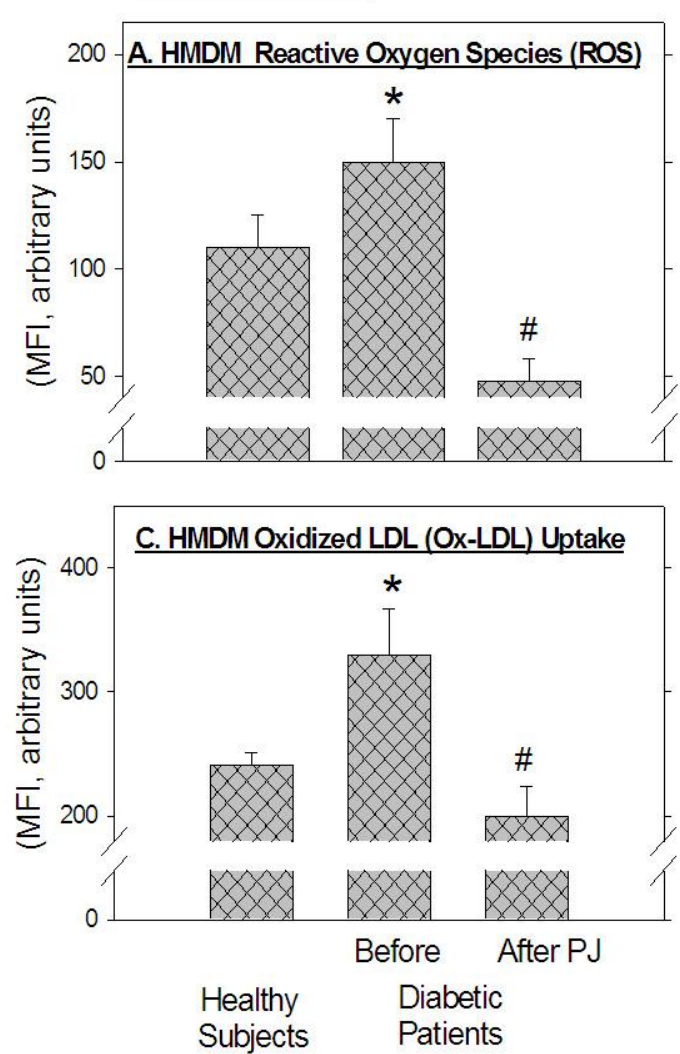

\section{CAS Patients}
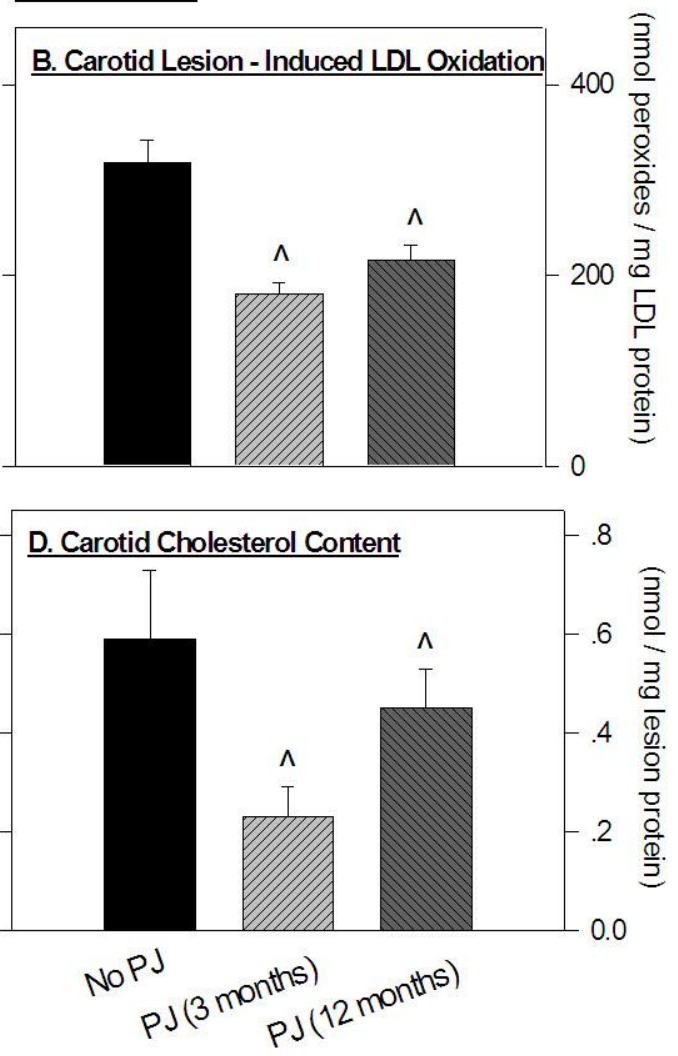

Figure 4. The anti-atherogenic effects of PJ consumption on HMDM from diabetic patients, and on carotid lesions from patients with CAS.

A and C: Monocytes were isolated from the blood of two healthy subjects and from three patients with type 2 diabetes before and after 3 months of PJ consumption ( $50 \mathrm{mLper}$ day). The monocytes were differentiated into macrophages in the presence of RPMI medium containing $10 \%$ autologous serum. After 7 days in culture the amount of ROS (A) and the uptake of Ox-LDL $(20 \mu \mathrm{g}$ of protein $/ \mathrm{mL})$ labeled with FITC by the cells (C) were determined. Results are given as mean \pm SEM. ${ }^{*} P<0.01$ diabetic patients' HMDM versus healthy subjects' HMDM, ${ }^{\#} P<0.01$ diabetic patients' HMDM after PJ consumption versus diabetic patients' HMDM before PJ consumption.

B and D: Lesions were collected from seven patients with CAS after endartherectomy and from two patients who consumed $\mathrm{PJ}$ for 3 and 12 months and had to undergo endartherectomy during the study, due to clinical deterioration. B: Lesions $(0.3 \mathrm{~g})$ were incubated with LDL (100 mg of protein $/ \mathrm{L})$ in PBS for 20 hours at $37^{\circ} \mathrm{C}$. The extent of LDL oxidation was measured by the lipid peroxide assay. Lesion-mediated oxidation of LDL was calculated by subtracting the values obtained in control LDL (incubation with no lesion) from those obtained after LDL incubation with the lesions. Three determinations were done on each lesion. D: The amount of cholesterol was measured in the lesion homogenates. Results represent mean $\pm S E M .{ }^{\wedge} P<0.01$ (carotid lesions after $P J$ consumption versus carotid lesions, no PJ). 
Figure $4 \mathrm{C}$ ), as was shown in vitro in J774A.1 macrophages. 46

The carotid lesions from CAS patients who consumed PJ contained also less cholesterol (Figure 4D). This could be related to the reduction in the amount of Ox-LDL and thus in Ox-LDL uptake, and also to PJ-induced increment in PON1, since PON1 was shown in vitro to inhibit macrophage cholesterol biosynthesis. 47 In addition, PJ can directly attenuate cholesterol biosynthesis by the cells, as was previously shown. ${ }^{6}$ The reduction in lesion cholesterol levels after PJ consumption could also result from stimulation of HDL-mediated cholesterol efflux by PON1.48

\section{ANTI-ATHEROGENICITY OF STATIN TREATMENT IN COMBINATION WITH POMEGRANATE}

Statins therapy made a significant health benefit, mainly in cardiovascular protection, 49 by improving the symptoms of atherosclerosis development. $5^{5}$ Statins are potent inhibitors of HMGCoA-reductase (the rate-limiting enzyme in cholesterol biosynthesis ${ }^{51}$ ), and they possess minor antioxidative properties. ${ }^{2}$ However, statins have also deleterious sideeffects when taken at high dosages for a long period of time. 53

Phytosterols, which encompass plant sterols and stanols, are steroid compounds similar in their structure to cholesterol.54 The richest sources of phytosterols are vegetable oils and products made from them. The most commonly occurring phytosterols in human diet are $\beta$-sitosterol, campesterol, and stigmasterol.54 Phytosterols consumption decreased serum cholesterol levels in dyslipidemic patients, as well as their cardiovascular risk. 55,56 Thus, phytosterols were suggested as an appropriate additional therapy to a low-dosage statin treatment.

We have recently57 analyzed in vitro the antiatherogenic effects on macrophage cholesterol biosynthesis rate, and on cellular oxidative stress, of the combination of simvastatin with punicalagin, or with a phytosterol ( $\beta$-sitosterol), or with PJ (that contains both of them 58,59$)$. Simvastatin $(15 \mu \mathrm{g} / \mathrm{mL})$ decreased the J774A.1 macrophage cholesterol biosynthesis rate by $42 \%$ as compared to control cells. The addition to the statin of either punicalagin (15 or $30 \mu \mathrm{M}$ ) or $\beta$-sitosterol (50 or $100 \mu \mathrm{M}$ ) increased the inhibitory effect of the statin up to $62 \%$ or $57 \%$, respectively. Similarly, the combination of PJ and simvastatin resulted in an inhibitory effect up to $59 \%$. While simvastatin inhibited the enzyme HMGCoA-reductase, punicalagin, $\beta$-sitosterol, and PJ inhibited macrophage cholesterol biosynthesis downstream to mevalonate. Simvastatin $(15 \mu \mathrm{g} / \mathrm{mL})$ also modestly decreased macrophage ROS formation by $11 \%$. In the presence of punicalagin ( 15 or $30 \mu \mathrm{M}$ ), however, a remarkable further inhibition was noted (by $61 \%$ or $79 \%$, respectively). Although $\beta$-sitosterol alone showed some pro-oxidant activity, the combination of simvastatin, $\beta$-sitosterol, and punicalagin clearly demonstrated a remarkable $73 \%$ reduction in ROS production. Similarly, simvastatin + PJ decreased the extent of ROS formation by up to $63 \%$.

These results suggest that PJ consumption by hypercholesterolemic patients together with treatment with a low dose of statins could lead to attenuation of macrophage foam cell formation and atherogenesis in these patients.

\section{CONCLUSIONS}

Pomegranate fruit polyphenols protect against lipid peroxidation in serum by direct interaction of pomegranate polyphenols with LDL, or indirectly by increasing serum PON1 stability (HDL association), as well as its catalytic activities, resulting in the hydrolysis of lipid peroxides. Moreover, PJ has a remarkable effect on macrophage and lesion atherogenicity. Pomegranate juice consumption decreased oxidative stress in macrophages and in atherosclerotic lesions, and the extent of Ox-LDL uptake by macrophages. This could be a direct antioxidant effect of PJ, or an indirect effect, by increasing HDLassociated PON1 as well as cellular PON2. Interestingly, the lesion cholesterol levels were decreased after PJ consumption. This could be related to the reduction in Ox-LDL uptake by macrophages, to PJ/PON1-induced inhibition of cholesterol biosynthesis, and to PON1 stimulation of HDL-mediated cholesterol efflux from arterial macrophages.

All these antioxidative and anti-atherogenic effects of pomegranate polyphenols were clearly demonstrated in vivo, in humans (healthy subjects, CAS patients, as well as diabetic patients). The preferred pomegranate product in terms of biological potency and consequent health benefits is PJ from the whole fruit (including the peel). Since the combination of antioxidants that exists in PJ can provide a wider range of free radical scavenging 
capacities than an individual antioxidant, clinical and nutritional studies in humans should be directed towards the use of combinations of several types of dietary antioxidants, as well as combinations of flavonoids together with other nutritional antioxidants, such as vitamin $\mathrm{E}$ or carotenoids. In addition, PJ can be beneficially used in combination with low-dose statins in hypercholesterolemic patients.

Finally, it is also important to identify populations suitable for antioxidant treatment, as antioxidants treatment may be beneficial only in subjects who are under excess oxidative stress.

\section{REFERENCES}

1. Langley P. Why a pomegranate? BMJ 2000;321:1153. Full Text

2. Gil MI, Tomas-Barberan FA, Hess-Pierce B, et al. Antioxidant activity of pomegranate juice and its relationship with phenolic composition and processing. J Agric Food Chem 2000;48:4581-9. Full Text

3. Ben Nasr C, Ayed N, Metche M. Quantitative determination of the polyphenolic content of pomegranate peel. Z Lebensm Unters Forsch 1996;203: 374. Full Text

4. Tzulker R, Glazer I, Bar-Ilan I, Holland D, Aviram M, Amir R. Antioxidant activity, polyphenol content, and related compounds in different fruit juices and homogenates prepared from 29 different pomegranate accessions. J Agric Food Chem 2007;55: 9559-70. Full Text

5. Cerdá B, Llorach R, Cerón JJ, Espín JC, TomásBarberán FA. Evaluation of the bioavailability and metabolism in the rat of punicalagin, an antioxidant polyphenol from pomegranate juice. Eur J Nutr 2003;42:18-23. Full Text

6. Cerdá B, Cerón JJ, Tomás-Barberán FA, Espín JC. Repeated oral administration of high doses of the pomegranate ellagitannin punicalagin to rats for 37 days is not toxic. J Agric Food Chem 2003;51:3493501. Full Text

7. Seeram NP, Lee R, Heber D. Bioavailability of ellagic acid in human plasma after consumption of ellagictannins from pomegranate (Punica granatum L.) juice. Clin Chim Acta 2004;348:63-8. Full Text

8. Perez-VicenteA, Gil-Izquierdo A, Garcia-VigueraC. In vitro gastrointestinal digestion study of pomegranate juice phenolic compounds, anthocyanins, and vitamin C. J Agric Food Chem 2002;50:2308. Full Text
9. Basu A, Penugonda K. Pomegranate juice: a hearthealthy fruit juice. Nutr Rev 2009;67:49-56. Full $\underline{\text { Text }}$

10. Aviram M, Dornfeld L, Kaplan M. Pomegranate juice flavonoids inhibit low-density lipoprotein oxidation and cardiovascular diseases: studies in atherosclerotic mice and in humans. Drugs Ex Clin Res 2002;28:49-62.

11. Sumner MD, Elliott-Eller M, Weidner G, et al. Effects of pomegranate juice consumption on myocardial perfusion in patients with coronary heart disease. Am J Cardiol 2005;96:810-14. Full Text

12. Aviram M, Rosenblat M, Gaitini D, et al. Pomegranate juice consumption for 3 years by patients with carotid artery stenosis reduces common carotid intima-media thickness, blood pressure and LDL oxidation. Clin Nutr 2004;23:423-33. Full Text

13. Davidson MH, Maki KC, Dicklin MR, et al. Effects of consumption of pomegranate juice on carotid intimamedia thickness in men and women at moderate risk for coronary heart disease. Am J Cardiol 2009;104: 936. Full Text

14. Mayr M, Kiechl S, Tsimikas S, et al. Oxidized lowdensity lipoprotein autoantibodies, chronic infections, and carotid atherosclerosis in a populationbased study. J Am Coll Cardiol 2006;47:2436-43. Full Text

15. Basarici I, Altekin RE, Demir I, et al. Associations of isoprostanes-related oxidative stress with surrogate subclinical and angiographic measure of atherosclerosis. Coron Artery Dis 2007;18:615-20. Full Text

16. Liu SX, Hou FF, Guo ZJ, et al. Advanced oxidation protein products accelerate atherosclerosis through promoting oxidative stress and inflammation. Arterioscler Thromb Vasc Biol 2006;26:1156-62. Full $\underline{\text { Text }}$

17. Polidori MC, Pratico D, Parente B, et al. Elevated lipid peroxidation biomarkers and low antioxidants status in atherosclerotic patients with increased carotid or iliofemoral intima media thickness. J Invest Med 2007;55:163-7. Full Text

18. Aviram M, Dornfeld L, Rosenblat $\mathrm{M}$, et al. Pomegranate juice consumption reduces oxidative stress, atherogenic modifications to LDL, and platelet aggregation: studies in humans and in atherosclerotic apolipoprotein E-deficient mice. Am J Clin Nutr 2000;71:1062-76.

19. Rosenblat M, Hayek T, Aviram M. Anti-oxidative effects of pomegranate juice (PJ) consumption by diabetic patients on serum and on macrophages. Atherosclerosis 2006;187:368-76. Full Text 
20. Gaidukov L, Tawfik DS. High affinity, stability, and lactonase activity of serum paraoxonase PON1 anchored on HDL with ApoA-I. Biochemistry 2005; 44:11843-54. Full Text

21. Fuhrman B, Volkova N, Aviram M. Paraoxonase 1 (PON1) is present in postprandial chylomicrons. Atherosclerosis 2005;180:55-61. Full Text

22. Rosenblat M, Aviram M. Paraoxonases role in the prevention of cardiovascular diseases. Biofactors 2009;35:98-104. Full Text

23. Mackness B, Davies GK, Turkie W, et al. Paraoxonase status in coronary heart disease. Are activity and concentration more important than genotype? Arterioscler Thromb Vasc Biol 2001;21:1451-7. Full Text

24. Harangi M, Seres I, Magyar MT, et al. Association between human paraoxonase 1 activity and intimamedia thickness in subjects under 55 years of age with carotid artery diseases. Cerebrovasc Dis 2008; 25:122-8. Full Text

25. Aviram M, Rosenblat M, Bisgaier CL, Newton RS, Primo-Parmo SL, La Du BN. Paraoxonase inhibits high-density lipoprotein oxidation and preserves its functions. A possible peroxidative role for paraoxonase. J Clin Invest 1998;101:1581-90. Full Text

26. Rozenberg O, Shih DM, Aviram M. Paraoxonase 1 (PON1) attenuates macrophage oxidative status: studies in PON1 transfected cells and in PON1 transgenic mice. Atherosclerosis. 2005;181:9-18. Full Text

27. Rozenberg O, Rosenblat M, Coleman R, Shih DM, Aviram M. Paraoxonase (PON1) deficiency is associated with increased macrophage oxidative stress: studies in PON1-knockout mice. Free Radic Biol Med. 2003;34:774-84. Full Text

28. Aviram M, Hardak E, Vaya J, et al. Human serum paraoxonases (PON1) Q and R selectively decrease lipid peroxides in human coronary and carotid atherosclerotic lesions: PON1 esterase and peroxidaselike activities. Circulation 2000;101: 2510-17. Full $\underline{\text { Text }}$

29. Khateeb J, Gantman A, Kreitenberg AJ, Aviram M, Fuhrman B. Paraoxonase 1 (PON1) expression in hepatocytes is upregulated by pomegranate polyphenols: a role for PPAR-gamma pathway. Atherosclerosis 2010;208:119-25. Full Text

30. Aviram M, Rosenblat M, Billecke S, et al. Human serum paraoxonase (PON 1) is inactivated by oxidized low density lipoprotein and preserved by antioxidants. Free Radic Biol Med 1999;26:892-904. Full Text

31. Rosenblat M, Karry R, Aviram M. Paraoxonase 1 (PON1) is a more potent antioxidant and stimulant of macrophage cholesterol efflux, when present in HDL than in lipoprotein-deficient serum: relevance to diabetes. Atherosclerosis 2006;187:74-81. Full Text

32. Rock W, Rosenblat M, Miller-Lotan R, Levy AP, Elias M, Aviram M. Consumption of wonderful variety pomegranate juice and extract by diabetic patients increases paraoxonase 1 association with high-density lipoprotein and stimulates its catalytic activities. J Agric Food Chem 2008;56:8704-13. Full Text

33. Fuhrman B, Volkova N, Aviram M. Pomegranate juice polyphenols increase recombinant paraoxonase1 binding to high-density lipoprotein: studies in vitro and in diabetic patients. Nutrition 2010;26:359-66. Full Text

34. Aviram M, Dornfeld L. Pomegranate juice consumption inhibits serum angiotensin converting enzyme activity and reduces systolic blood pressure. Atherosclerosis 2001;158:195-8. Full Text

35. Lynn A, Hamadeh H, Leung WC, Russell JM, Barker ME. Effects of pomegranate juice supplementation on pulse wave velocity and blood pressure in healthy young and middle-aged men and women. Plant Foods Hum Nutr 2012;67:309-14. Full Text

36. Mathew AS, Capel-Williams GM, Berry SE, Hall WL. Acute effects of pomegranate extract on postprandial lipaemia, vascular function and blood pressure. Plant Foods Hum Nutr 2012;67:351-7. Full Text

37. Stowe CB. The effects of pomegranate juice consumption on blood pressure and cardiovascular health. Complement Ther Clin Pract 2011;17:113-15. Full Text

38. Tiwari RL, SinghY, Barthwal MK. Macrophages: an elusive yet emerging therapeutic target of atherosclerosis. Med Res Rev 2008;28:483-544. Full Text

39. Lusis AJ. Atherosclerosis. Nature 2000;404:233-41. Full Text

40. Aviram M, Rosenblat M. Oxidative Stress in Cardiovascular Disease: Role of Oxidized Lipoproteins in Macrophage Foam Cell Formation and Atherosclerosis. In: Fuchs J, Podda M, Packer L., eds. Redox-Genome Interactions in Health and Disease. New York: CRC Press; 2003:57-90. Full $\underline{\text { Text }}$

41. Steinberg D. Low density lipoprotein oxidation and its pathobiological significance. J Biol Chem 1997; 272:20963-6. Full Text

42. Aviram M, Rosenblat M. Paraoxonases 1, 2 and 3, oxidative stress, and macrophage foam cell formation during atherosclerosis development. Free Radic Biol Chem 2004;37:1304-16. Full Text

43. Shiner M, Fuhrman B, Aviram M. Macrophage paraoxonase 2 ( $\mathrm{PON} 2$ ) expression is up-regulated by 
pomegranate juice phenolic anti-oxidants via PPAR gamma and AP-1 pathway activation. Atherosclerosis 2007;195:313-21. Full Text

44. Rosenblat M, Draganov D, Watson CE, Bisgaier CL, La Du BN, Aviram M. Mouse macrophage paraoxonase 2 activity is increased whereas cellular paraoxonase 3 activity is decreased under oxidative stress. Arterioscler Thromb Vasc Biol 2003;23:468-74. Full $\underline{\text { Text }}$

45. Altenhöfer S, Witte I, Teiber JF, et al. One enzyme, two functions: PON2 prevents mitochondrial superoxide formation and apoptosis independent from its lactonase activity. J Biol Chem 2010;285:24398-403. Full Text

46. Fuhrman B, Volkova N, Aviram M. Pomegranate juice inhibits oxidized LDL uptake and cholesterol biosynthesis in macrophages. J Nutr Biochem 2005; 16:570-6. Full Text

47. Rozenberg O, Shih DM, Aviram M. Human serum paraoxonase 1 decreases macrophage cholesterol biosynthesis: possible role for its phospholipase-A2like activity and lysophosphatidylcholine formation. Arterioscler Thromb Vasc Biol 2003;23:461-7. Full $\underline{\text { Text }}$

48. Rosenblat M, Gaidukov L, Khersonsky O, et al. The catalytic histidine dyad of high density lipoproteinassociated serum paraoxonase-1 (PON1) is essential for PON1-mediated inhibition of low density lipoprotein oxidation and stimulation of macrophage cholesterol efflux. J Biol Chem 2006;281:7657-65. Full Text

49. Bożentowicz-Wikarek M, Kocełak P, Smertka M, Olszanecka-Glinianowicz M, Chudek J. Effectiveness of lipid-lowering therapy with statins for secondary prevention of atherosclerosis - guidelines vs. reality. Pharmacol Rep 2012;64:377-85.

50. Davignon J. Pleiotropic effects of pitavastatin. Br J Clin Pharmacol 2012;73:518-35. Full Text
51. Lupattelli G, Siepi D, De Vuono S, et al. Cholesterol metabolism differs after statin therapy according to the type of hyperlipemia. Life Sci 2012;90:846-50. Full Text

52. Sobal G, Sinzinger H. Effect of simvastatin on the oxidation of native and modified lipoproteins. Biochem Pharmacol 2005;70:1185-91. Full Text

53. Goldfine AB. Statins: is it really time to reassess benefits and risks? N Engl J Med 2012;366:1752-5. Full Text

54. Ostlund RE Jr. Phytosterols in human nutrition. Annu Rev Nutr 2002;22:533-49. Full Text

55. Moruisi KG, Oosthuizen W, Opperman AM. Phytosterols/stanols lower cholesterol concentrations in familial hypercholesterolemic subjects: a systematic review with meta-analysis. J Am Coll Nutr 2006;25:41-8.

56. Malinowski JM, Gehret MM. Phytosterols for dyslipidemia. Am J Health Syst Pharm 2010;67: 1165-73. Full Text

57. Rosenblat M, Volkova N, Aviram M. Pomegranate phytosterol ( $\beta$-sitosterol) and polyphenolic antioxidant (punicalagin) addition to statin, significantly protected against macrophage foam cells formation. Atherosclerosis 2013;226:110-17. Full Text

58. Van Elswijk DA, Schobel UP, Lansky EP, Irth H, Van Der Greef J. Rapid dereplication of estrogenic compounds in pomegranate (Punica granatum) using on-line biochemical detection coupled to mass spectrometry. Phytochemistry 2004;65:233-41. Full Text

59. Mori-Okamoto J, Otawara-Hamamoto Y, Yamato H, Yoshimura H. Pomegranate extract improves a depressive state and bone properties in menopausal syndrome model ovariectomized mice. J Ethnopharmacol 2004;92:93-101. Full Text 